\title{
A NOVEL FRONTAL SINUS INDEX PROTOCOL FOR GENDER DETERMINATION: A RETROSPECTIVE LATERAL CEPHALOMETRIC STUDY FOR EGYPTIANS
}

\author{
Sarah Mohammed Kenawy*, Mohamed Taha T. Ellabban** and Ali Fahd Fadel ${ }^{* * *}$
}

\begin{abstract}
Aim: To develop and assess a new protocol proposed for sex determination using frontal sinus index measured from two dimensional lateral cephalometric radiographs.

Materials \& Methods: The frontal sinus index was measured by two experienced radiologists and one experienced orthodontist on two hundred and one cephalometric scans (93 males \& 108 females). The maximum height and width of the sinus were measured then the height to width ratio was calculated. The mean height to width ratio of the sinus were tabulated and subjected to statistical analysis to determine the correlation to gender.
\end{abstract}

Results: There was a high statistically significant difference between the frontal sinus index measured between males and females groups.

Conclusion: Frontal sinus index measured on lateral cephalometric radiographs by the developed protocol could be an indicator for gender identification in Egyptians and a larger sample multicentric study is recommended.

KEYWORDS: Frontal Sinus Index, Lateral Cephalometric Radiography, Gender, Forensic Medicine

\section{INTRODUCTION}

Forensic odontology plays an important part in personal identification especially when postmortem changes invalidate the use of other approaches like fingerprint ${ }^{[1]}$. Although forensic dentistry goes long back in history, there are evolutions in the used techniques ${ }^{[2]}$.
Because forensic and anthropological research is based on a set of physical characteristics that are unique to every individual, unique anatomical landmarks can be used ${ }^{[3]}$. Dental imaging is considered a reliable objective way of data collection that can be used in identification so that it can be used in forensic dentistry ${ }^{[4]}$.

\footnotetext{
* Lecturer, Department of Oral and Maxillofacial Radiology, Faculty of Dentistry, Cairo University, Egypt.

** Lecturer, Department of Orthodontics and Dento-Facial Orthopedics, Faculty of Dentistry, Assiut University, Egypt.

*** Lecturer, Department of Oral and Maxillofacial Radiology, Faculty of Oral and Dental Medicine, South Valley University, Egypt.
} 
The history of diagnostic radiology began in 1895 when the first radiograph was made by Professor Roentgen and it is only three years later when first radiographic autopsy examination was presented ${ }^{[5]}$. Lateral cephalometric radiographs are an invaluable tool in identification of gender as they expose architectural and morphological details of the skull on a single radiographic image. The equipment needed for lateral cephalometric imaging is widely available and the technique is of low cost, easy to perform, reproducible and can be employed in any special training for the forensic inspect ${ }^{[6]}$.

Gender determination is a crucial factor in forensic medicine. In addition, Dental imaging is a corner stone in forensic odontology and sinus radiography may be used for identification of gender [7]. Frontal sinus is one of the imaged anatomical landmarks that is unique in shape and may be described as fingerprint-like individuality, hence frontal sinus index (FSI) can be used in personal identification for forensic purposes ${ }^{[8]}$. Previous studies measured FSI and correlated it to gender are not only limited and contradicting but also lack a reliable standardized way of application ${ }^{[9-11]}$.

Considering the aforementioned background, this study aimed to develop and assess new protocols for reliable sex determination using frontal sinus index measured from two dimensional lateral cephalometric radiographs.

\section{MATERIAL AND METHODS}

This retrospective study was conducted on 201 lateral cephalometric scans for patients who had been referred to a private radiology center in Cairo, Egypt. The scans were selected from its database with patients' age chosen to be above 20 years old. The randomly selected patients were 93 males and 103 females. Radiographs of patients below 20 years of age, scans with artifacts, congenital malformations, agenesis, pathology affecting the frontal sinus and history of surgery in the anatomical area of interest were excluded.
All lateral cephalometric images were performed by Pax-i3D Green cephalometric machine (Vatech, Gyeonggi-do, Korea) on adult individuals using a standard imaging protocol performed by a licensed radiology technician. The obtained images were exported in a "Joint Photographic Experts Group" (JPEG) file format and transferred to personal computers then imported using Romexis 5.3 software (Planmeca, Helsinki, Finland). The scans were analyzed after calibration by two radiologists and one orthodontist with more than 10 years of experience and doctorate degree holders. A vertical line connecting the most upper point with the lowest point of the frontal sinus was measured and the horizontal line was drawn from the most anterior point crossing perpendicular to the vertical line reaching the corresponding posterior border of the frontal sinus. The frontal sinus index is the ratio of the measured height to the measured width. The scores obtained were tabulated with the respective gender for each patient and subjected to statistical analysis. Data in the present study were presented as mean, standard deviation (S.D.), and Confidence Interval (C.I.) values. Statistical analysis was performed with IBM SPSS Statistics Version 22 for Windows. The significance level was set at $\mathrm{P} \leq 0.05$. Kappa agreement test was used for interobserver agreement.

The proposed novel standardized approach started by same amount of magnification which was done using actual pixels tool (1:1) from view toolbar. Calibration was done using the calibration tool from measure toolbar in the software interface. A line was drawn connecting $30 \mathrm{~mm}$ of the ruler from bottom upward then the reference length was typed (figure 1).

The outline of the sinus was drawn through the draw curve tool and color-coded red. A green color-coded rectangle was drawn tangent with the highest and lowest points ( $\mathrm{H}$ and $\mathrm{L}$ ) of drawn border. A yellow color-coded vertical line was drawn tangent with the most anterior point of the drawn border (A). The vertical measurement line was drawn connecting $\mathrm{H}$ and $\mathrm{L}$ points and was blue 


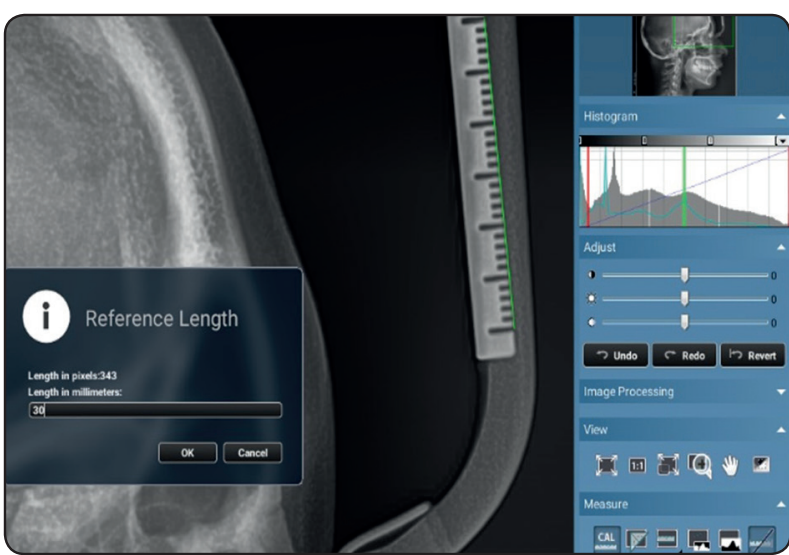

Fig. (1): The calibration line (green line on ruler side), calibration box, the used magnification tool (view toolbar) and calibration tool (measure toolbar)

colored. The horizontal line was started at the A point and pass perpendicular to the vertical line till reaching the most posterior drawn border and was white colored. To ensure that the horizontal line is perpendicular to the vertical line a 90-degree angle was measured and was black colored (figure 2).

\section{RESULTS}

Total of 201 lateral cephalometric scans were analyzed for measuring FSI as gender identification tool in 93 males \& 108 females. Results of ShapiroWilk tests showed that data was not normally

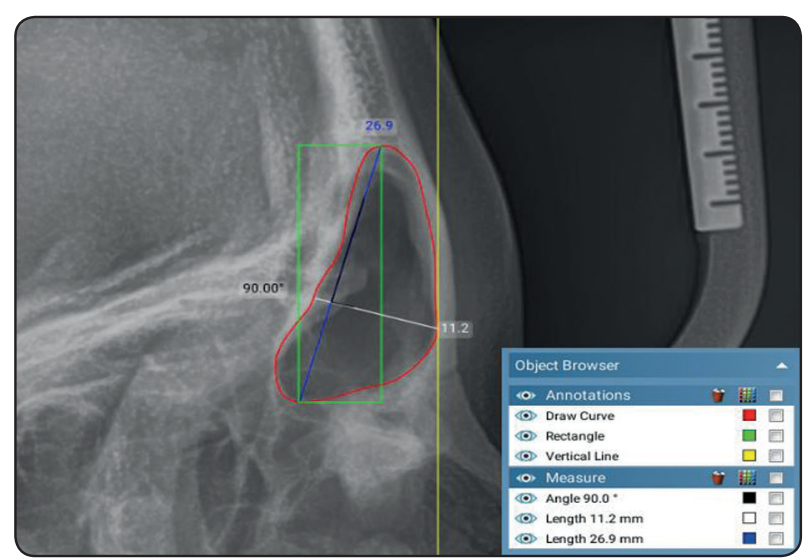

Fig. (2): The color-coded standard protocol.

distributed so non-parametric Mann-Whitney test was used for compassion between both groups. Table (1) gives the descriptive statistics of the frontal sinus index as mean, standard deviation and standard error mean.

The statistical test of data showed a statistically significant mean difference of FSI between male and female groups where the mean frontal sinus index of female group was larger significantly than that of male group as shown in table (2). Kappa agreement test showed weak inter-observer agreement.

TABLE (1): Descriptive statistics of the frontal sinus index in our study

\begin{tabular}{|l|l|r|r|r|r|}
\hline & Sex & $\mathrm{N}$ & \multicolumn{1}{|c|}{ Mean } & \multicolumn{1}{c|}{ Std. Deviation } & \multicolumn{1}{c|}{ Std. Error Mean } \\
\hline \multirow{2}{*}{ Index } & Male & 93 & 2.6443 & .85618 & .08878 \\
\cline { 2 - 6 } & Female & 108 & 3.4867 & .93655 & .09012 \\
\hline
\end{tabular}

TABLE (2): Statistical test for data of our study

\begin{tabular}{|l|r|r|r|r|r|r|}
\hline & \multirow{2}{*}{$\begin{array}{c}\text { Mean } \\
\text { Difference }\end{array}$} & \multirow{2}{*}{$\begin{array}{c}\text { Std. Error } \\
\text { Difference }\end{array}$} & \multicolumn{2}{|c|}{$95 \%$ CI of the Difference } & \multirow{2}{*}{$Z^{\dagger}$} & \multirow{2}{*}{ P value } \\
& & & Lower & Upper & & \\
\hline Male Index - Female index & -.84231 & 0.12736 & -1.09346 & -0.59117 & -6.771 & $0.000^{*}$ \\
\hline
\end{tabular}




\section{DISCUSSION}

Gender determination is an essential part in forensic dentistry which could be determined by various dental related approaches including tooth morphology prints of the lip or palatal rugae, and measurements of paranasal sinuses ${ }^{[12]}$. The unique shape of frontal sinus was the motivator for this research. This was confirmed early by researchers like Asherson in 1965 who despite of doing his work on twins found that frontal sinus was always different ${ }^{[13]}$. Quatrehomme et al positively identified two forensic cases using frontal sinus and in one of them helped in arresting of the murderer ${ }^{[14]}$.

Adult cases were chosen because the frontal sinus is considered stable after development of the individual ${ }^{[15,16]}$. Frontal sinus is invisible radiographically until around six years of age and continues pneumatization through puberty till ceasing at about 20 years of age when its size and shape become stable ${ }^{[17,18]}$. That's why patients below the age of 20 were excluded.

Digital oral and maxillofacial imaging is an easy way for morphometric analysis of sinuses ${ }^{[19]}$. This work was based on simple, cost effective methodology for gender estimation based on frontal sinus index measured on lateral cephalometric radiograph. Although maxillofacial imaging had evolved from conventional two-dimensional techniques to advanced three dimensional technologies, lateral cephalometry still playing a predominant role for skull analysis for its low dose, cost effectiveness, availability, and reliable accuracy ${ }^{[12,20,21]}$. The problem with 2D imaging was that any minute changes in the angulation and position relation between the object, the receptor and the machine might alter the anatomical characteristics ${ }^{[22]}$. To overcome this problem, the ratios were used because of being considered more reliable regarding factors like geometric distortion ${ }^{[10]}$. Also, calibration was done before any measurements. During calibration, the ruler was measured for a large length which decreases the margin of error due to inaccurate positioning of the start or end point of the line and to avoid deviation the line was drawn in match with the ruler border.

For the proposed novel standardization protocol, the rectangle was used as a simple and accurate way for ensuring parallelism of the upper and lower tangent reference lines making a point of contact with the highest and lowest borders so that the vertical line connecting them was standardized. Also, the rectangle could be adjusted to point at the needed areas. The software vertical line tool allowed a standard vertical line without any difficulties and was used as the anterior tangent reference line. The only difficult part was making the horizontal line perpendicular to the vertical line and ensuring this by the 90 -degree angle.

The exact distinct anatomical borders of the frontal sinus might not be clear on 2D radiographs due to shot angle, distance and adjacent anatomy ${ }^{[22,}$ ${ }^{23]}$ which was expected to decrease the credibility of the research results and may be a reason for reduced interobserver agreement. Despite of that, statistical analysis for each observer revealed the same end results which gives credibility to the results. Also, this was the cause for choosing the horizontal line to be perpendicular to the vertical line as the most diverse sinus outline is located in the posterior border making decision on the widest area more challenging.

Another factor for differences in frontal sinus border detection which caused decreased interobserver agreement was that the left and right sinuses are developed independently with the end result of asymmetrical sinuses ${ }^{[24]}$ leading to diversity in shape that was aggravated by the superimposition of both of them in 2D images. Fortunately, even with operator dependent difference in shape detection, applying the same measuring protocol gave a highly statistically significant same results for each operator.

On the other hand, some studies showed diversity results from ours as ElBeshlawy \& Helaly who found that the FS index could only act as a poor 
sex predictor ${ }^{[11]}$. This might be due to their different methodology.

Our comparative results showed that the frontal sinus indices assessed on lateral cephalometric images were significantly higher in females. Similar to us, some comparable studies performed on adults as Ramaswamy et al found that the sinus index was significantly higher in females ${ }^{[10]}$. In this study, images were imported to another software so that patient data were not shown, and operators could be blinded during measuring phase.

\section{CONCLUSION}

Measuring the frontal sinus index by the proposed protocol is promising. Multicentric study is recommended to reach a solid evidence-based results. Also testing the reliability of the standardization protocol is recommended by applying it on multiple drawn sinus borders by multiple experienced radiologists.

\section{REFERENCES}

1. Singh, N. N., Gowhar, O., Ain, T. S., \& Sultan, S. (2014). Exploring trends in forensic odontology. Journal of clinical and diagnostic research: JCDR, 8(12), ZC28.

2. Balachander, N., Babu, N. A., Jimson, S., Priyadharsini, C., \& Masthan, K. M. K. (2015). Evolution of forensic odontology: An overview. Journal of pharmacy \& bioallied sciences, 7(Suppl 1), S176.

3. Veyre-Goulet, S. A., Mercier, C., Robin, O., \& Guérin, C. (2008). Recent human sexual dimorphism study using cephalometric plots on lateral teleradiography and discriminant function analysis. Journal of Forensic Sciences, 53(4), 786-789.

4. Jeddy, N., Ravi, S., \& Radhika, T. (2017). Current trends in forensic odontology. Journal of forensic dental sciences, 9(3), 115.

5. Manigandan T., Sumathy C., Elumalai M., Sathasivasubramanian S., \& Kannan A., Forensic radiology in dentistry, J Pharm Bioallied Sci. 2015 Apr; 7(Suppl 1): S260-S264.

6. Binnal, A., \& Yashoda Devi, B. (2012). Identification of Sex using Lateral Cephalogram: Role of Cephalofacial Parameters. Journal of Indian Academy of Oral Medicine and Radiology, 24, 280-283.
7. Khaitan, T., Kabiraj, A., Ginjupally, U., \& Jain, R. (2017). Cephalometric analysis for gender determination using maxillary sinus index: a novel dimension in personal identification. International journal of dentistry, 2017.

8. Kumar, A. P., Doggalli, N., \& Patil, K. (2018). Frontal sinus as a tool in identification. International Journal of Forensic Odontology, 3(2), 55.

9. Benghiac, A. G., Thiel, B. A., \& Haba, D. (2015). Reliability of the frontal sinus index for sex determination using CBCT. Rom J Leg Med, 23(4), 275-8.

10. Ramaswamy, P., \& Khaitan, T. (2014). Frontal sinus index-A new tool for sex determination. Journal of Forensic Radiology and Imaging, 2(2), 77-79.

11. ElBeshlawy, D. M., \& Helaly, Y. R. (2020). Frontal sinus index for sex estimation: Is it possible?. Forensic Imaging, 23, 200407.

12. Kanthem, R. K., Guttikonda, V. R., Yeluri, S., \& Kumari, G. (2015). Sex determination using maxillary sinus. Journal of forensic dental sciences, 7(2), 163.

13. Asherson, N. (1965). Identification by frontal sinus prints. HK Lewis \& Company Limited.

14. Quatrehomme, G., Fronty, P., Sapanet, M., Grévin, G., Bailet, P., \& Ollier, A. (1996). Identification by frontal sinus pattern in forensic anthropology. Forensic science international, 83(2), 147-153.

15. Prossinger, H., \& Bookstein, F. L. (2003). Statistical estimators of frontal sinus cross section ontogeny from very noisy data. Journal of Morphology, 257(1), 1-8.

16. Meschan, I. (1959). An atlas of normal radiographic anatomy. Academic Medicine, 34(10), 1055.

17. Tatlisumak, E., Ovali, G. Y., Asirdizer, M., Aslan, A., Ozyurt, B., Bayindir, P., \& Tarhan, S. (2008). CT study on morphometry of frontal sinus. Clinical Anatomy: The Official Journal of the American Association of Clinical Anatomists and the British Association of Clinical Anatomists, 21(4), 287-293.

18. Moore, K., \& Ross, A. (2017). Frontal sinus development and juvenile age estimation. The Anatomical Record, 300(9), 1609-1617.

19. Inal, M., Simsek, G., Kaya, A., \& Kilic, R. (2019). Morphometric Analysis of the Fronto-maxillary Sinuses in Adult Patients with Traumatic Septal Deviations. Current Medical Imaging, 15(2), 194-198. 
20. Sidhu, R., Chandra, S., Devi, P., Taneja, N., Sah, K., \& Kaur, N. (2014). Forensic importance of maxillary sinus in gender determination: a morphometric analysis from Western Uttar Pradesh, India. European Journal of General Dentistry, 3(1), 53.

21. Mathur, R. U., Mahajan, A. M., Dandekar, R. C., \& Patil, R. B. (2014). Determination of sex using discriminant function analysis in young adults of Nashik: a lateral cephalometric study. Journal of Advanced Medical and Dental Sciences Research, 2(1), 21-25.

22. Cameriere, R., Ferrante, L., Mirtella, D., Rollo, F. U., \& Cingolani, M. (2005). Frontal sinuses for identification: quality of classifications, possible error and potential corrections. Journal of forensic sciences, 50(4), 770-773.

23. Luo, H., Wang, J., Zhang, S., \& Mi, C. (2018). The application of frontal sinus index and frontal sinus area in sex estimation based on lateral cephalograms among Han nationality adults in Xinjiang. Journal of forensic and legal medicine, 56, 1-4.

24. Tehranchi, A., Motamedian, S. R., Saedi, S., Kabiri, S., \& Shidfar, S. (2017). Correlation between frontal sinus dimensions and cephalometric indices: A cross-sectional study. European journal of dentistry, 11(1), 64. 